\title{
Convective flow in the presence of a small obstacle: Symmetry breaking, attractors, hysteresis, and information
}

\author{
S. J. Bartlett ${ }^{*}$ and Y. L. Yung \\ Division of Geological and Planetary Sciences, California Institute of Technology, Pasadena, California 91125, USA \\ and Earth-Life Science Institute, Tokyo Institute of Technology, Tokyo 152-8550, Japan
}

(Received 27 June 2018; revised manuscript received 11 February 2019; published 4 March 2019)

\begin{abstract}
This work explores the stability and hysteresis effects that occur when a small sink of momentum is introduced into a heat-driven, two-dimensional convective flow. As per standard fluid mechanical intuition, the system minimizes work generation and dissipation when one component of momentum is extracted. However, when the sink absorbs all incoming momentum, the system configures itself such that one of the convection plumes aligns directly with the sink. This state is the most hydrodynamically stable, but it maximizes, rather than minimizes extracted mechanical work. Furthermore, in the case of only vertical momentum extraction, there are two attractors, with different stabilities. Numerical experiments involving slow variations of the horizontal momentum extraction show a clear history dependence. This hysteresis preserves information about the system's past states, and hence represents a primitive memory. The momentum sink can also be used to manipulate the horizontal position of the flow field, with potential applications in microfluidics and laminar convection systems. This simple system exhibits the phenomena of autocatalysis (during the initial growth of the convection plumes), negative feedback (the attractors are either fully or quasistable), memory, and elementary computation.
\end{abstract}

DOI: 10.1103/PhysRevE.99.033103

\section{INTRODUCTION}

This paper highlights some curious hydrodynamic features of natural convection, that arise when momentum is extracted from a finite area of the flow field. We present a series of numerical simulations, which exhibit either minimization or maximization of work generation, depending on which components of momentum are extracted. We also demonstrate the bistability, hysteresis, and resulting memory that occur when time variation of momentum extraction is applied to the system.

Natural convection of a single phase fluid is a fundamental example of self-organization in a nonequilibrium system. Above the critical value of thermal driving force (the dimensionless Rayleigh number), the quiescent, static state becomes unstable to perturbations. Warmer fluid parcels rise as they are displaced by colder parcels, which fall, and the system eventually exhibits an organized configuration of convection cells. The earliest research on this elegant phenomenon was carried out by Claude Bénard and Lord Rayleigh, after whom the process was named [1]. The coherent and structured nature of convection compel any physical scientist to seek a compact mathematical description, and Malkus was arguably the first to do so [2-4]. He found that a simple boundary layer analysis reveals a possible extremum behavior. Assuming the boundary temperatures of the system are held constant, the fluid appears to maximize its heat flux, subject to the constraints of finite system size, fluid thermal diffusivity, and other physical constants.

\footnotetext{
*sjb@gps.caltech.edu; https://www.gps.caltech.edu/
}

At high Rayleigh numbers, the flow shows chaotic, turbulent features (though suitably averaged transport properties become stationary), and the mathematical difficulties of deterministic chaos and nonlinearity prohibit a simple solution (e.g., [5]). This led researchers to seek relations that describe the coarse-grained, bulk features of such systems, and several have been proposed. In the middle of the 20th century, while exploring extremum principles to describe the large-scale features of atmospheric heat transport, Paltridge discovered that maximization of entropy production produced accurate predictions [6]. The so-called maximum entropy production (MEP) principle soon attracted considerable interest, and was found to successfully predict dynamic atmospheric characteristics of Earth, Mars, and Titan [7-9]. However, the same approach fails for Venus [10], and the principle cannot be derived from first principles (despite the failed attempt by Dewar [11]). Several authors have claimed that convection is an example of MEP [12-14]; however, it has now been shown that this principle is not generally applicable, due to the effects of different types of boundary conditions [15-20].

Alongside MEP, researchers in Earth and atmospheric science previously appealed to a more firmly established thermodynamic principle: the heat engine. Since convection generates fluid kinetic energy through the dissipation of a thermal gradient, the analogy between convection and heat engines is enticing [21-28]. A natural hypothesis is then that convection maximizes its heat-to-work conversion efficiency (with a natural upper limit from Carnot's theorem). If true, this would be an elegant example of a natural phenomenon exhibiting a behavior that engineers strive to produce in their designed machines.

However, there are difficulties in appropriately choosing variables for a convective, dissipative heat engine [29,30], 
and there are several key differences between an ideal Carnot engine, and convective heat transport. Firstly, convection exhibits dissipative, irreversible phenomena, primarily viscous dissipation of kinetic energy. The majority of this dissipation occurs in the boundary layer between the ground and the bulk fluid. Atmospheric convection is not quasistatic or reversible (though in some cases, this may serve as a first approximation). Secondly, assuming a closed system, there is no concept of useful work extraction in a pure convective system. The purpose of an engine is to extract mechanical energy from a heat flux between thermal reservoirs. In the case of convection, all generated work is dissipated in the same system (in steady state, generation of kinetic energy is balanced by dissipation). The definition of efficiency is thus questionable, because all work that is produced stays within the system. If it were possible to place a wind turbine or momentum sink in a convective flow, one could readily make use of the heat engine analogy, because some fraction of the generated kinetic energy would be extracted and removed.

This direct analogy was one of the motivations for the present work. The following question was posed: when a sink of momentum is placed within a convective flow, how much mechanical work can be extracted, and how does this vary with the size of the momentum sink and other parameters? We were motivated by whether the natural process of convection can come close to the upper limit of the Carnot cycle. Basic hydrodynamic intuition would suggest that when an obstacle is placed within a flow, the flow will minimize its interaction with the obstacle by avoiding it. Thus one might expect that extracting work from convection constitutes an optimization problem, wherein positioning and sizing of the momentum sink must be carefully tuned such that flow avoidance is minimized. Previous work involving convection and flow obstacles focused upon systems with arrays of obstacles and porous media, or heated vertical boundaries (rather than horizontal, as in the present work), primarily for the design of heat exchangers and similar devices [31-40]. The isolated effects of single momentum sinks has received less attention, and previous efforts were aimed at the engineering of man-made systems. In contrast, our primary goal was to answer the scientific question of whether convection is an ideal converter of heat to work.

A further objective of this study was to gain deeper insight into the nonlinear dynamical and thermodynamic properties of convection. In recent decades there has been increasing interest in the mechanisms by which physical systems store and process information $[41,42]$. One might not expect a convective system to retain historical information, instead its state seems more likely to be a pure function of its boundary conditions. However, memory effects in atmospheric convection have been observed [43], and strange attractors have also been described for several hydrodynamic systems [44-47]. Nonetheless, the mechanisms by which information is created and destroyed in such systems remain to be fully understood.

Results presented herein demonstrate that convection exhibits a complex attractor landscape with multiple states of different stabilities. Given that the simulations did not delve into the turbulent regime, this complexity is clearly just a hint of what lies at higher Rayleigh numbers. The system can also be manipulated using time-varying momentum sinks. This susceptibility and multistability allows the system to store information about past conditions in its present condition, with implications for the spontaneous emergence of memory and information processing. From a fluid control perspective, these properties permit external manipulation of the flow, with potential engineering applications.

The following sections present results from a series of numerical simulations. Section II describes the standard Rayleigh-Bénard system without obstacles, Sec. III presents a system with a sink of horizontal momentum, Sec. IV presents a system with a vertical sink, and Sec. V a system with a sink of total momentum. We then turn to variations in extracted work as a function of time and sink size in Sec. VI. Section VII explores a simple mechanism by which hysteresis and memory emerge. We conclude and suggest further directions in Secs. VIII and IX.

\section{UNPERTURBED SYSTEM}

This work focuses upon natural convection of single-phase fluids. We use the conventional Boussinesq approximation, which assumes that density variations are sufficiently small to be ignored, except in the gravitational body force term. This assumption of incompressibility leads to a simple continuity equation:

$$
\nabla \cdot \mathbf{v}=0,
$$

where $\mathbf{v}=u \hat{i}+v \hat{k}$ is the nondimensional fluid velocity. Conservation of momentum leads to the following momentum equation:

$$
\frac{\partial \mathbf{v}}{\partial t}+\mathbf{v} \cdot \nabla \mathbf{v}+\nabla P=\frac{v}{\chi} \nabla^{2} \mathbf{v}+\frac{v}{\chi} \frac{\beta g_{0} \Delta T^{\prime} \delta^{3}}{\nu \chi} T \hat{k} .
$$

This reveals the two dimensionless groups that govern the fluid dynamical behavior: the thermal driving force or Rayleigh number $\mathrm{Ra}=\beta g_{0} \Delta T^{\prime} \delta^{3} / v \chi$, and the ratio of viscous to thermal diffusivity $\operatorname{Pr}=v / \chi$. Finally, the advectiondiffusion equation describes the transport of internal energy:

$$
\frac{\partial T}{\partial t}+\mathbf{v} \cdot \nabla T=\chi \nabla^{2} T .
$$

The response of the system to the imposed boundary conditions is quantified by the dimensionless heat flux or Nusselt number: $\mathrm{Nu}=Q H / \chi \Delta T^{\prime}$, where $Q$ is the average heat flux through the system, and $\chi \Delta T^{\prime} / H$ is the diffusive heat flux given the temperature gradient, system size, and thermal diffusivity.

For this investigation, we made use of a simple computational fluid dynamics technique known as the lattice Boltzmann model, which can accurately recreate the thermohydrodynamics of single-phase convection. The method numerically solves the nonequilibrium Boltzmann equation for an incompressible fluid. The momentum equation and the advection-diffusion equation shown above can then be derived from the lattice Boltzmann equation [48-52]. The Prandtl number was fixed at $\operatorname{Pr}=1$, and the numerical domain consisted of $512 \times 256$ grid cells. This grid resolution was more than sufficient to resolve the features of the laminar flows that were simulated. 


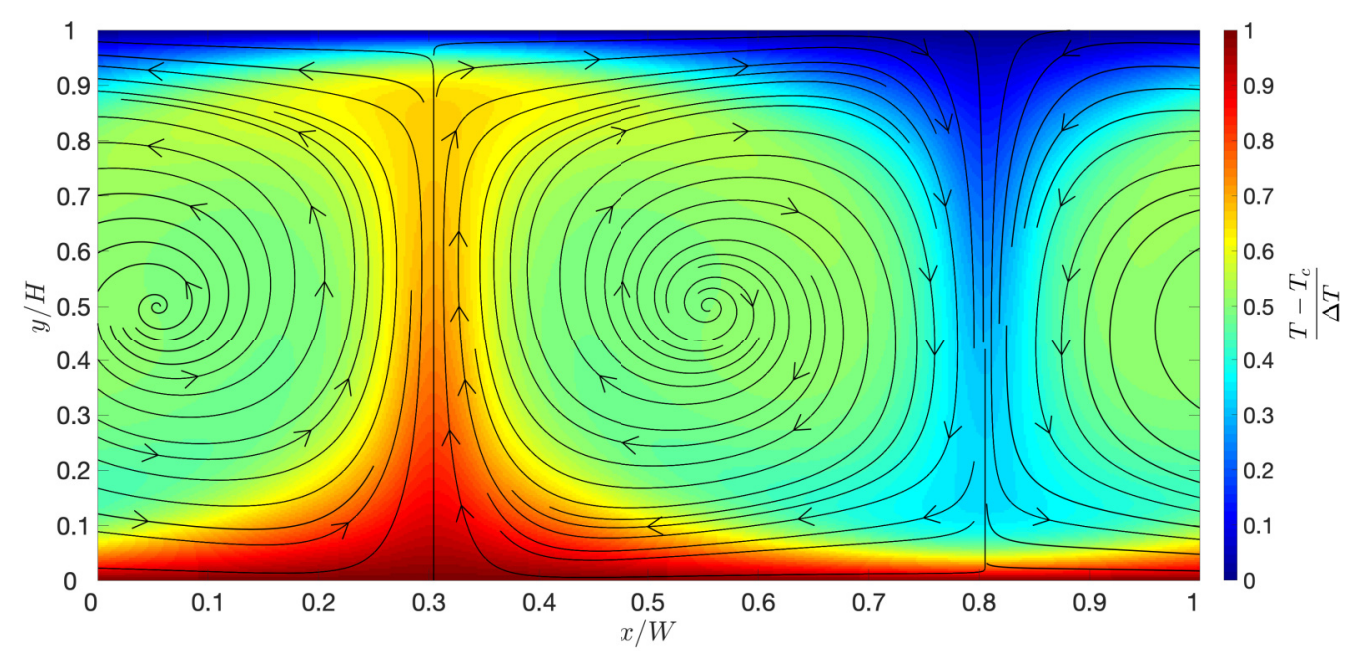

(a)

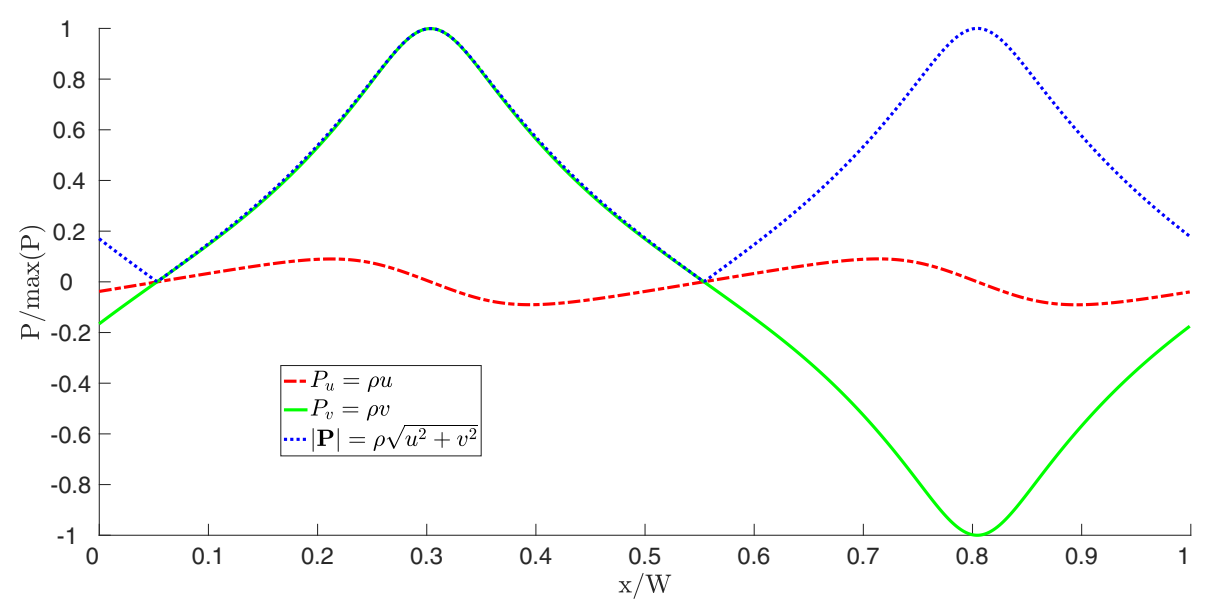

(b)

FIG. 1. Flow structure of a convective fluid driven at a Rayleigh number of $\mathrm{Ra}=10^{5}$. (a) Flow and temperature fields (dimensionless temperature scale is shown on the right). (b) Components and magnitude of the fluid momentum at the horizontal midplane of the system. Red dot-dashed line corresponds to horizontal momentum, solid green to vertical momentum, and dotted blue to total momentum magnitude. Values are normalized by the maximum momentum magnitude. $\rho$ is fluid density.

The generic flow structure for laminar convection (without a momentum sink) is shown in Fig. 1(a) (note that periodic boundary conditions are used in the horizontal direction throughout the present work). Figure 1(b) shows the momentum components and magnitude as measured along the horizontal midplane of the system. This illustrates the periodic structure of the flow field, with extrema in vertical momenta in the centers of the two convection plumes. Note also the small but nonzero horizontal momentum along this midplane [red dot-dashed line in Fig. 1(b)].

This system has translational symmetry in the horizontal direction: its stability (Hamiltonian) does not depend on the horizontal phase shift of the flow field. We quantify this horizontal phase shift with the following definition:

$$
\phi=\frac{1}{W} \min _{i \in C}\left\{\left|x_{o}-x_{v_{i}}\right|\right\},
$$

where $W$ is the system width, $x_{o}$ is the horizontal position of the obstacle, $x_{v_{i}}$ is the horizontal location of the center of vortex $i$, and $C$ is the set of vortices (also see Fig. 2 for a graphical definition).

\section{HORIZONTAL MOMENTUM SINK}

We now introduce a sink of momentum to the system. This sink consisted of a small region $(2 \times 2$ grid cells $)$ in which a certain fraction of momentum was removed at each time step. These fractions are denoted by $m_{u}$ and $m_{v}$ for horizontal and vertical momentum, respectively. That fraction of fluid motion of the relevant component(s) was eliminated using an imposed body force. In all simulations the sink is placed in the center of the domain $\left[x_{\text {sink }}, y_{\text {sink }}\right]=[W / 2, H / 2]$. The flow field was free to adjust itself in response to the extraction of momentum by the sink. In the first case, only horizontal momentum was extracted: $\left[m_{u}, m_{v}\right]=[1,0]$. A typical steady-state configuration for such a system is shown in Fig. 3.

As expected, the presence of the momentum sink has broken the horizontal translational symmetry of the free 


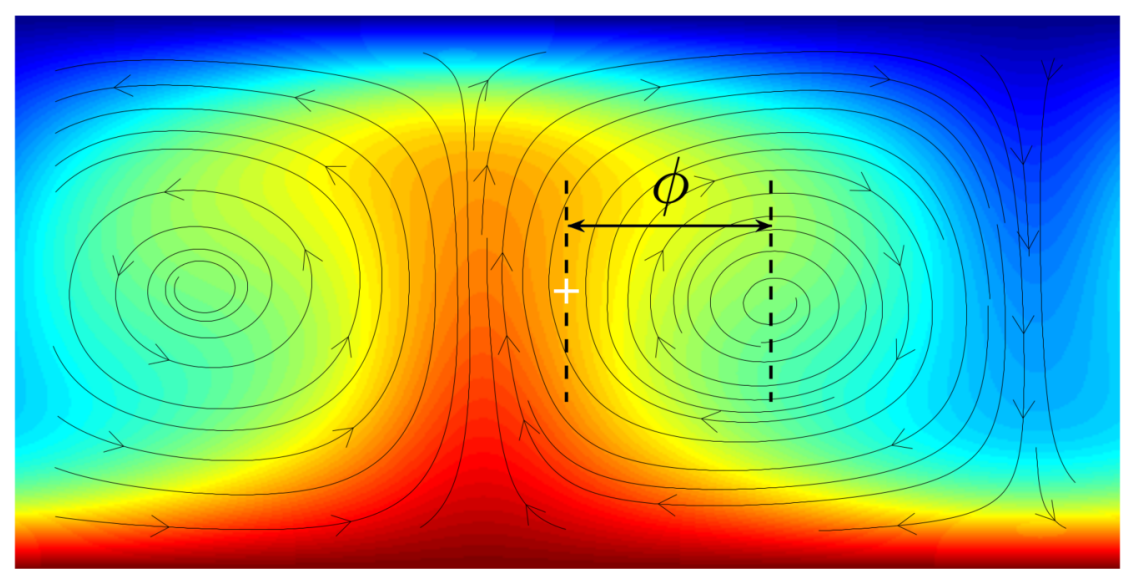

FIG. 2. Graphical definition of the phase parameter $\phi$, which measures the minimum distance between a convection cell center and the momentum sink, that is shown with white crosshairs.

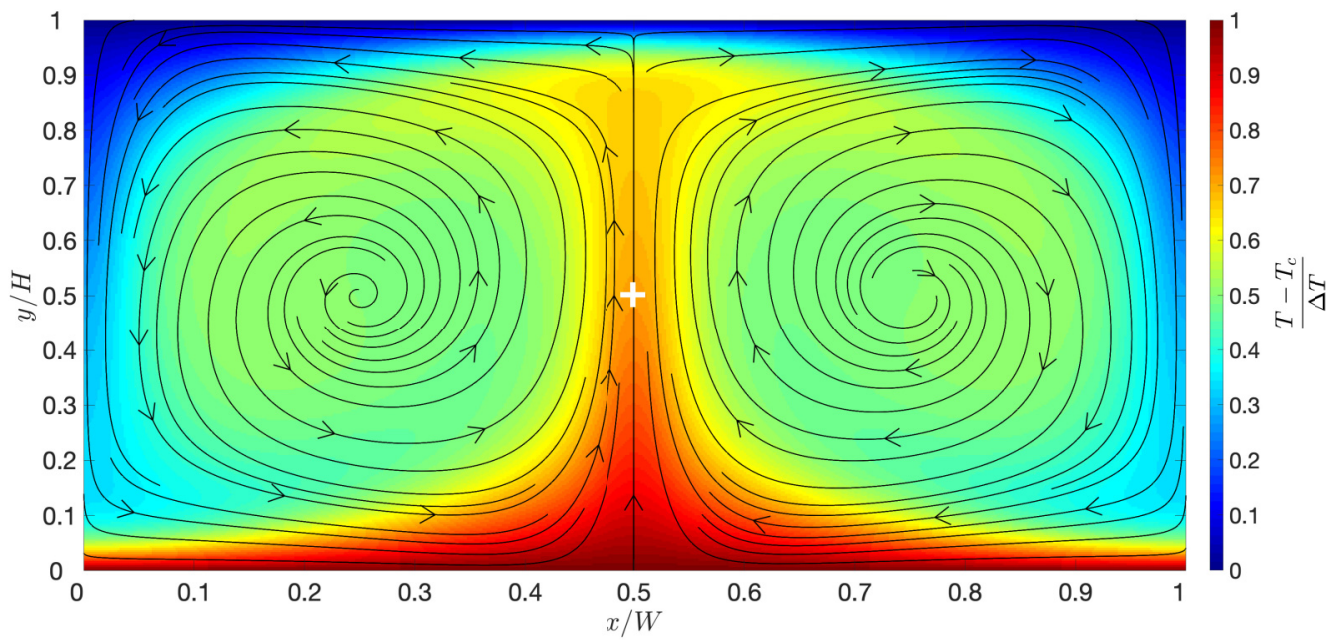

(a)

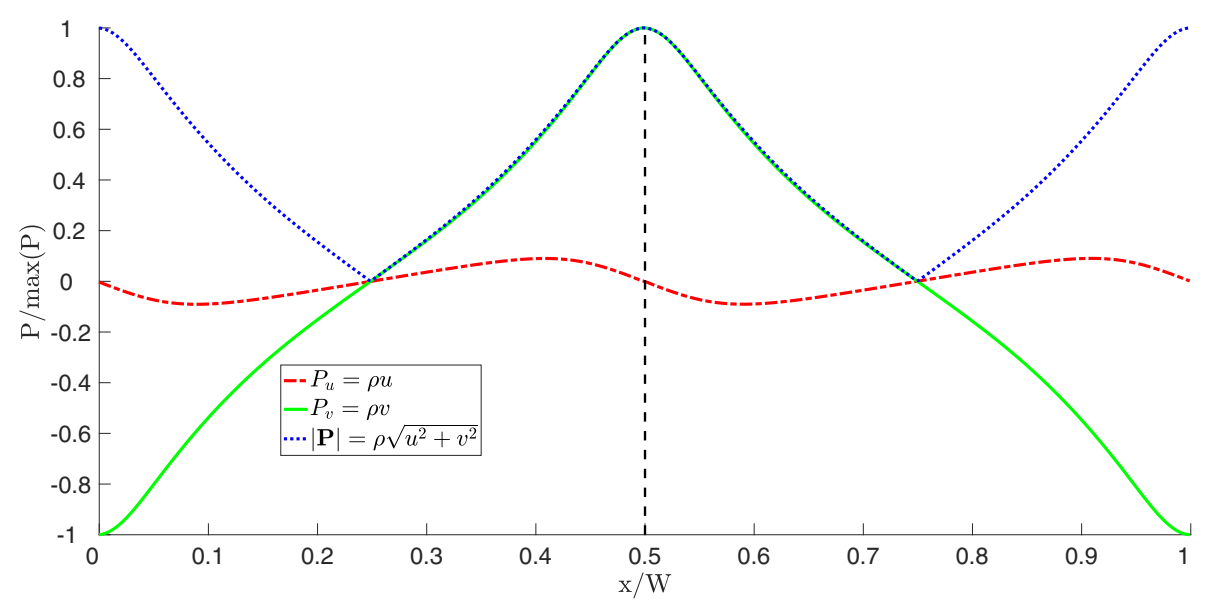

(b)

FIG. 3. Typical flow structure of a convective fluid driven at a Rayleigh number of $\mathrm{Ra}=10^{5}$ in the presence of a small sink of horizontal momentum placed at $[x, y]=[W / 2, H / 2]$ (the white crosshairs show its location, its size is only $\sim 0.7 \%$ of the system height). (a) Flow and temperature fields (dimensionless temperature scale is shown on the right). (b) Components and magnitude of the fluid momentum at the horizontal midplane of the system. Red dot-dashed line corresponds to horizontal momentum, solid green to vertical momentum, and dotted blue to total momentum magnitude. Values are normalized by the maximum momentum magnitude, which was essentially invariant to the type of momentum sink present. 


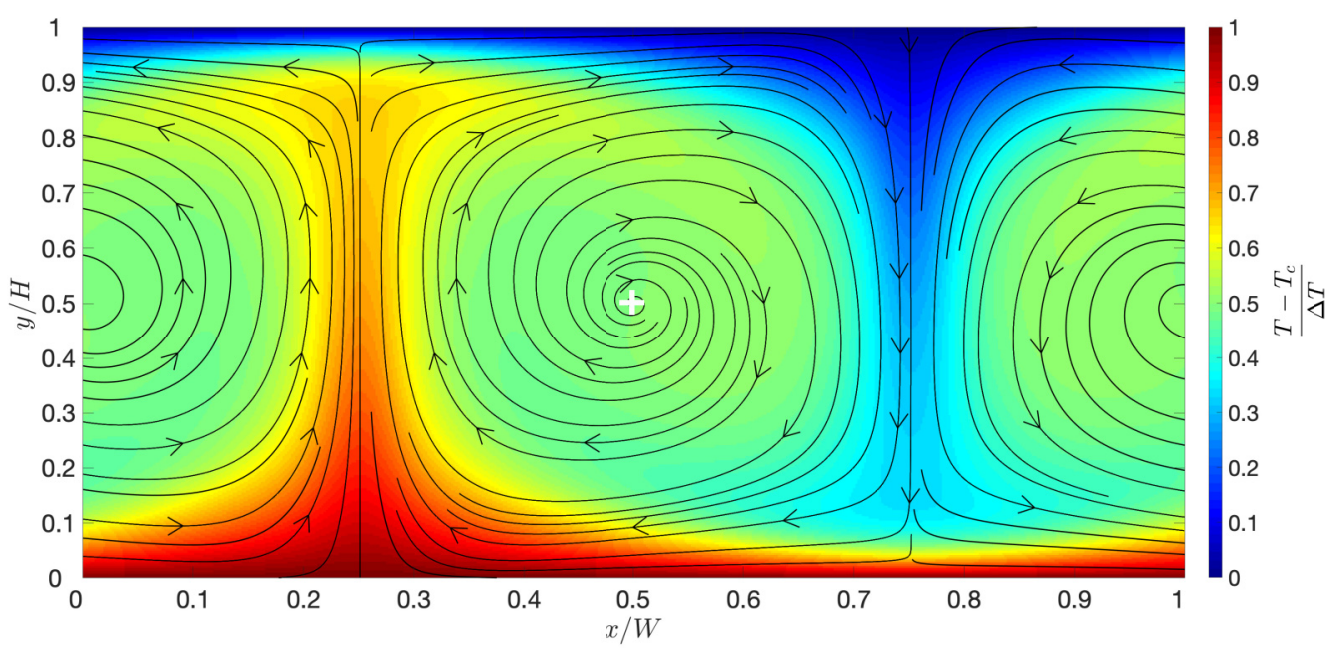

(a)

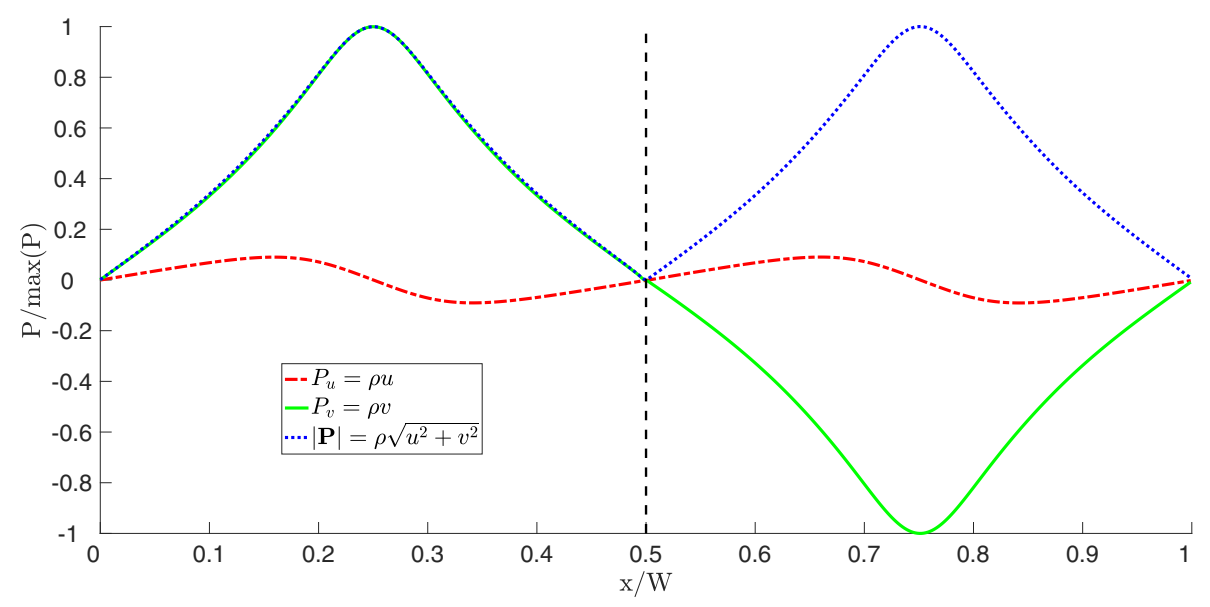

(b)

FIG. 4. Typical flow structure of a convective fluid driven at a Rayleigh number of $\mathrm{Ra}=10^{5}$ in the presence of a small sink of vertical momentum placed at $[x, y]=[W / 2, H / 2]$ (the white crosshairs show its location, its size is only $\sim 0.7 \%$ of the system height). (a) Flow and temperature fields (dimensionless temperature scale is shown on the right). (b) Components and magnitude of the fluid momentum at the horizontal midplane of the system. Red dot-dashed line corresponds to horizontal momentum, solid green to vertical momentum, and dotted blue to total momentum magnitude. Values are normalized by the maximum momentum magnitude, which was essentially invariant to the type of momentum sink present.

convection system. The horizontal phase shift has adjusted so that the momentum sink lies within the upwelling convection plume: $\phi=1 / 4$. The system settled into alignment with the downwelling plume in approximately half of the simulations, as would be expected (see Fig. 9 for illustrations of both attractors). The deciding factor between these two stable states was simply the random initial conditions.

It is clear from the momentum structure in Fig. 3(b) that these two configurations (alignment with either convection plume) are stable to perturbations. This is because any horizontal fluctuation in the plume position is corrected by the fact that horizontal flows are antisymmetric and focusing about the plume centers. Even though the convection cell centers are locations of vanishing momentum, and hence could be attractors, they are unstable to perturbations. Despite the fact that the horizontal momentum vanishes at $x=W / 4,3 W / 4$, it has a positive gradient at these points [red curve in Fig. 3(b)].
Minimum dissipation (work extraction) corresponds to the state of greatest dynamical stability in this case, as would be expected. Viewed from the perspective of convection as a heat engine, a horizontal momentum sink system produces minimal work (in fact none at all), making such a system useless as an engine.

\section{VERTICAL MOMENTUM SINK}

A vertical momentum sink produces different stable attractors, as shown in Fig. 4. In this case, the two stable states are alignments with either vortex center (see Fig. 10 for illustrations of both attractors). Any perturbation away from this configuration would induce a shear stress from the gradient of vertical velocity as a function of horizontal position. This shear stress would act to bring the convection cell back to a position where the momentum sink lies at its 


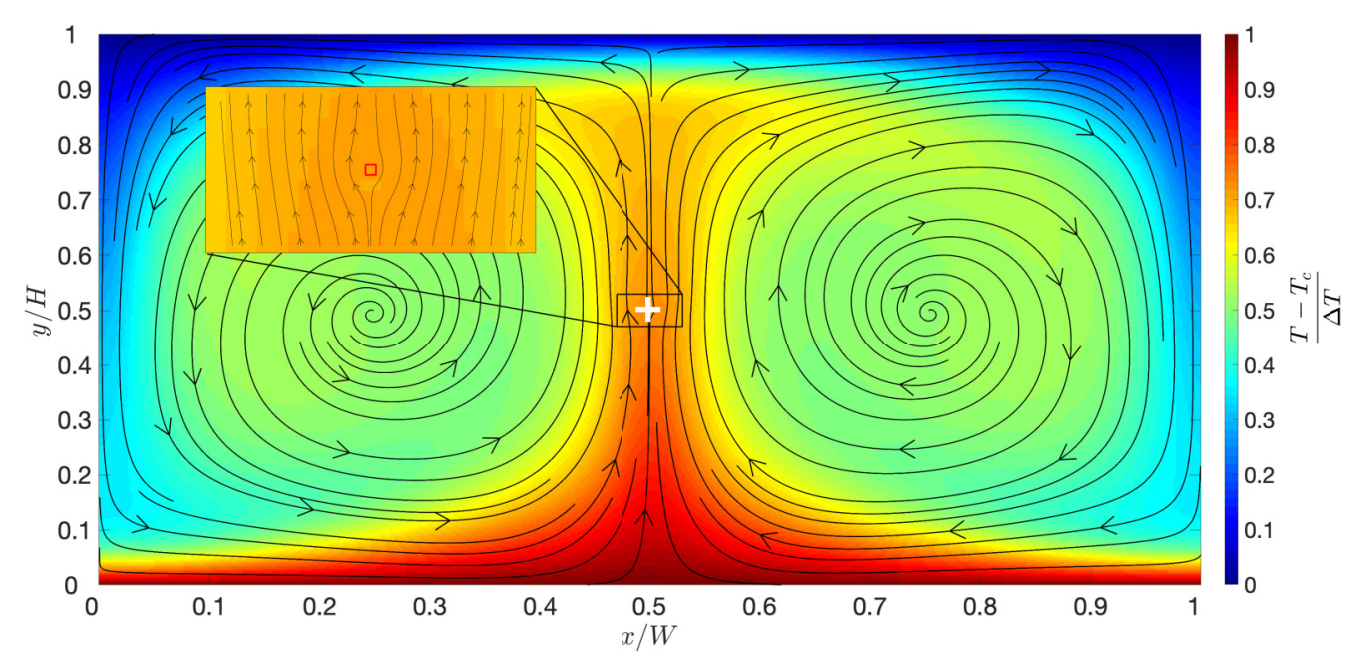

(a)

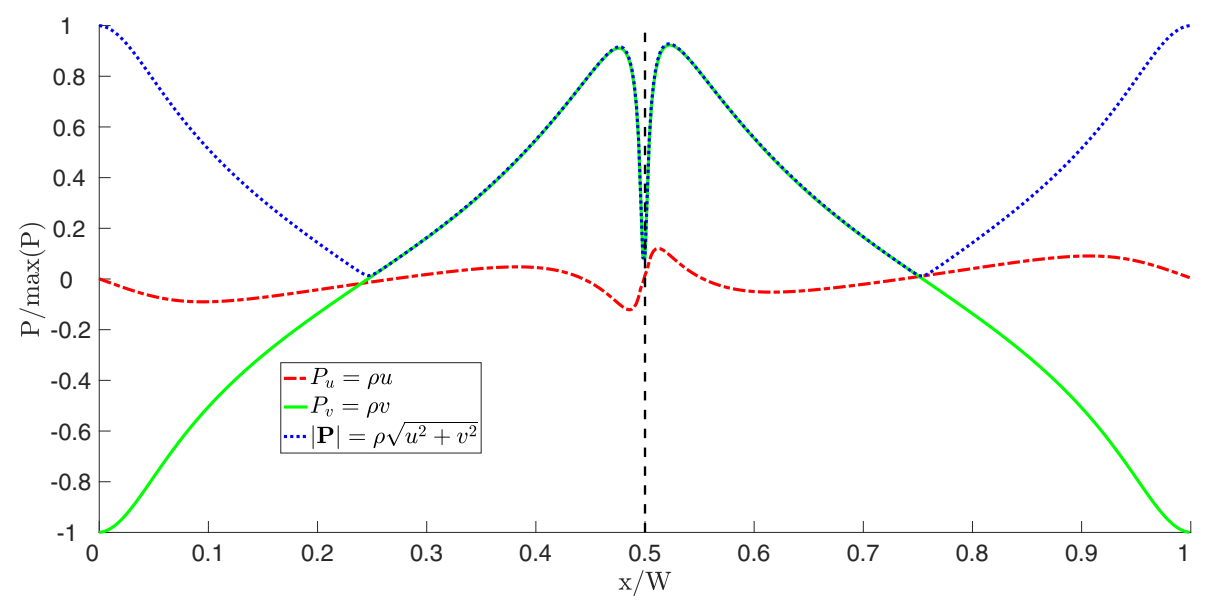

(b)

FIG. 5. Typical flow structure of a convective fluid driven at a Rayleigh number of Ra $=10^{5}$ in the presence of a small sink of total momentum placed at $[x, y]=[W / 2, H / 2]$ (the white crosshairs show its location; its size is only $\sim 0.7 \%$ of the system height). (a) Flow and temperature fields (dimensionless temperature scale is shown on the right). Inset figure shows the local flow around the obstacle, with the red box indicating the area from which all momentum is extracted. (b) Components and magnitude of the fluid momentum at the horizontal midplane of the system. Red dot-dashed line corresponds to horizontal momentum, solid green to vertical momentum, and dotted blue to total momentum magnitude. Values are normalized by the maximum momentum magnitude, which was essentially invariant to the type of momentum sink present.

center. As was the case with the horizontal sink, the stable configurations are also those of minimum work extraction by the sink.

In contrast to the horizontal case, there is an additional pair of stable states for the vertical sink system (see Fig. 11 for illustrations of these two additional attractors). These two states are the same as those for the horizontal sink (alignment with either convection plume). They are not as common as the vortex attractors, since it is rare that the system spontaneously settled upon them ( $\sim 10 \%$ occurrence rate), and they exhibited oscillations, especially at higher Rayleigh numbers. However, moving from the plume state to the stable attractor of the convection cell required a certain degree of deformation of the flow structure. The energetic barrier associated with this deformation is what allowed the less stable states to occasionally occur. For the plume attractors, the local lateral deflection of fluid due to the vertical sink actually created an effective sink of horizontal momentum leading to similar attractive behavior as seen in Secs. III and V. This bistability is explored in greater detail in Sec. VII.

\section{TOTAL MOMENTUM SINK}

For the most common attractors of the previous two sections, the system adjusts itself into states that are the most dynamically stable, and these states are also those of least work extraction (minimum internal dissipation). When a total momentum sink is placed in the system, it behaves in a manner that is at first sight surprising, as shown in Fig. 5 (also see Fig. 12 for all attractors).

The flow arranges itself such that the point of momentum extraction coincides with a maximum of vertical momentum 
(either convection plume), despite the presence of a minimum in total momentum at the center of either convection cell. Why do the system's stable states not coincide with those of minimum work output? There is a simple hydrodynamic symmetry explanation for this result. Aligning either plume with the momentum sink is stable to perturbations because the upstream horizontal momentum always acts to keep the plume centered upon the sink. This self-alignment automatically solves one dimension of the convective heat engine optimization problem: one does not need a strategy for the placement of momentum sinks in the flow, since the flow automatically migrates toward such sinks, when both components of momentum are extracted.

All systems considered in the present work made use of periodic boundary conditions. If the system instead had noslip vertical walls at either side, it would only exhibit a single attractor state of two convection cells with a plume at center. This is due to the strong geometric constraint of a rectangular boundary with a 2:1 aspect ratio: The most stable state in such a system is one in which convection cells that are of similar size to the vertical dimension fill the system.

The periodic boundary conditions remove this constraint, but permit the system to communicate with itself horizontally. This is somewhat analogous to having an infinite horizontal array of systems, each with an obstacle at their centers. We performed additional tests in which the aspect ratio of the system was increased (increased $W$ at constant $H$ ), hence decreasing the influence of the periodic boundary conditions. It was found that the attractive (repulsive) behaviors described in the previous sections still arise.

We also experimented with nonperiodic boundaries (noslip vertical walls). In this case a large aspect ratio is required to recover the attractive (repulsive) effects. It appears that an aspect ratio of $\sim 7: 1$ is sufficient to diminish the finite-size constraints imposed by the vertical walls. The hydrodynamic influence of the obstacle has to compete with the influence of the vertical walls in this case. Thus larger obstacles are better able to produce the attractive (repulsive) effects. Further experiments along these lines are currently being performed.

\section{WORK OUTPUT AND OSCILLATIONS IN FLOW STRUCTURE}

In the following section we consider trends in the extracted work as a function of Rayleigh number and obstacle size. We make use of the standard reference velocity $U=\left(\beta g_{0} \Delta T H\right)^{1 / 2}$, reference time $\tau=H / U$, and reference kinetic energy density $w=\rho_{0} U^{2} / 2$.

The total kinetic energy extracted from the sink was equal to

$$
\begin{aligned}
W= & \frac{V}{2} \sum_{i, j \in S} \rho_{i, j}\left[u_{i, j}^{2}+v_{i, j}^{2}-\left\{\left(\left[1-m_{u}\right] u_{i, j}\right)^{2}\right.\right. \\
& \left.\left.+\left(\left[1-m_{v}\right] v_{i, j}\right)^{2}\right\}\right] \\
= & \frac{V}{2} \sum_{i, j \in S} \rho_{i, j}\left[m_{u} u_{i, j}^{2}\left(2-m_{u}\right)+m_{v} v_{i, j}^{2}\left(2-m_{v}\right)\right],
\end{aligned}
$$

where $V$ is the volume of a grid cell, $S$ is the set of sink cells, $i$ and $j$ are grid indices, $\rho_{i, j}$ is fluid density, and $u_{i, j}$ and $v_{i, j}$ are the fluid velocity components before application of the body force. Note that the sink removes a fraction $m_{u}$ and $m_{v}$ of the fluid's horizontal and vertical momenta (velocities), respectively. The extracted work $W$ can be normalized by $W_{0}=w n_{S} V$, where $n_{S}$ is the number of grid cells in the sink area.

We can also define an "engine efficiency" $\eta=W / Q_{a}$, where $Q_{a}$ is the total heat flux at the lower boundary of the system. Hydrodynamic intuition would suggest that this

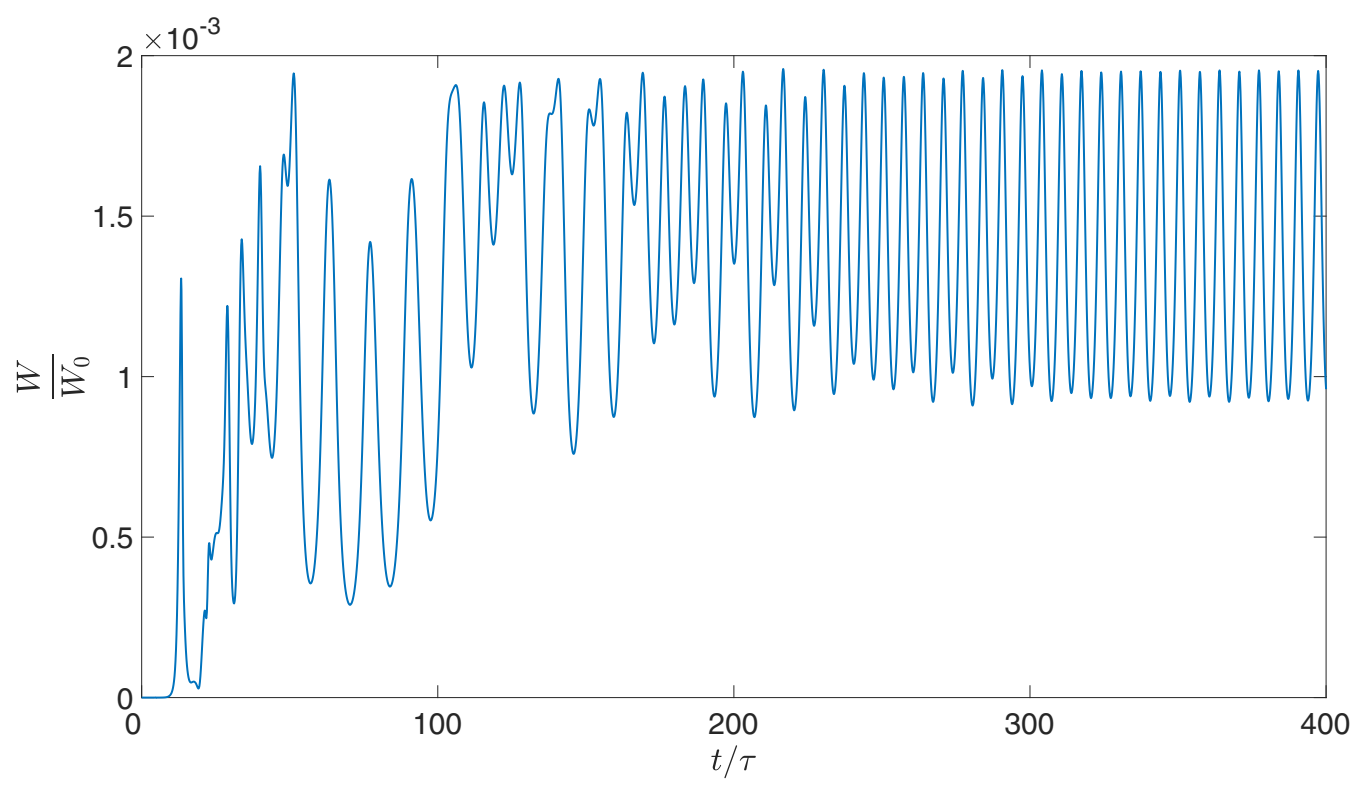

FIG. 6. Work extracted from the total momentum sink as a function of time during a simulation with $\mathrm{Ra}=2 \times 10^{5}$. Time is normalized by the reference time $\tau$, and work is normalized by the characteristic kinetic energy $W_{0}$. The maxima in the oscillatory part of the curve correspond to the plume pointing directly at the obstacle, and the minima correspond to the plume pointing to either side of the obstacle. 


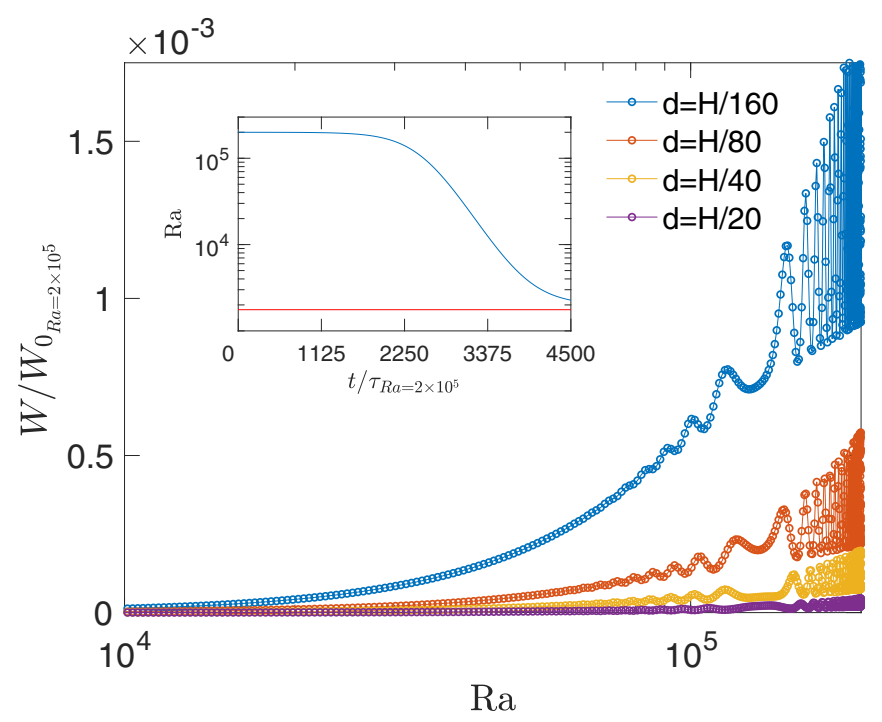

FIG. 7. Kinetic energy extracted from the total momentum sink as a function of Rayleigh number. Curves were derived from simulations in which the Rayleigh number was slowly varied, as shown in the inset figure that displays Rayleigh number as a function of time (blue line). Time units are normalized by the characteristic timescale of the initial Rayleigh number, i.e., that corresponding to $\mathrm{Ra}=$ $2 \times 10^{5}$. The red line in the inset figure corresponds to the critical Rayleigh number of $\mathrm{Ra}_{c} \approx 1760$. In the main figure, the four curves illustrate the influence of obstacle diameter $d$, given as a fraction of the system height $H$, upon the extracted work. As the obstacle radius becomes a larger fraction of the system height, the extracted work decreases. Work values are normalized by the reference kinetic energy $W_{0_{\mathrm{Ra}=2 \times 10^{5}}}$, corresponding to the initial Rayleigh number of $2 \times 10^{5}$, and adjusted for each sink size (see beginning of Sec. VI).

measure is likely to be very small for the following reason. In the absence of obstacles and in steady state, the generation rate of kinetic energy is balanced by viscous dissipation (primarily at the no-slip walls). For incompressible flows at low Mach number, such as the laminar flows in the present work, the viscous dissipation is normally omitted due to its negligible magnitude. Given that the viscous dissipation is equal to the kinetic energy generation rate, we can surmise that the energetic magnitude of fluid kinetic energy is dwarfed by the magnitude of the heat flux through most convective systems, including those of the present work. Approximate values for $\eta$ were found to be less than $1 \%$, far from the upper bound of the Carnot cycle. They are not quantitatively accurate because heat exchanges due to pressure-volume work and viscous dissipation are not included in the modeling framework employed here (see below).

As mentioned in Sec. I, previous work has suggested that atmospheric and/or mantle convection may come close to the Carnot limit [21-28]. For this to be the case would require turbulent flows at very high Rayleigh number to exhibit significantly greater kinetic energy generation rates per unit heat flux than laminar flows. Testing this will require highresolution direct numerical simulations that can accurately incorporate viscous dissipation, compressibility effects, and heat exchanges due to pressure-volume work. This is an endeavor that we are currently undertaking.

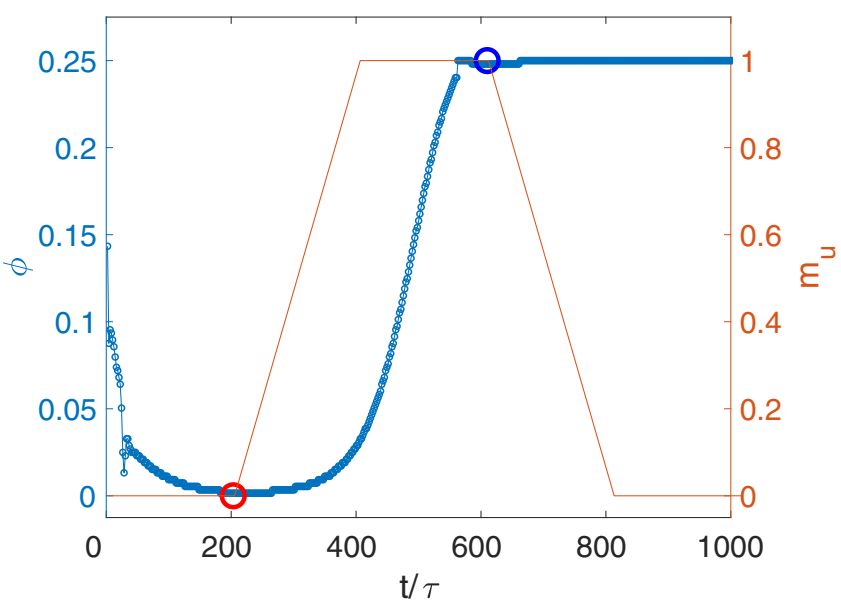

(a)

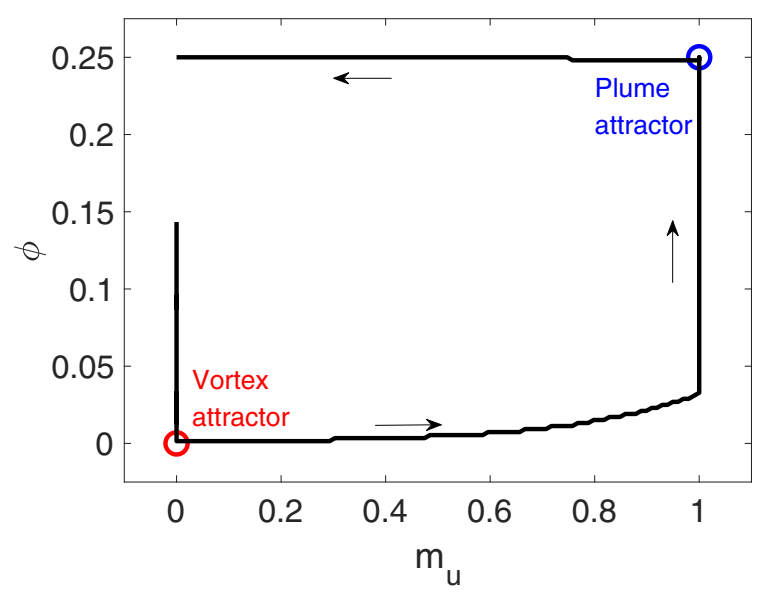

(b)

FIG. 8. Phase-space behavior for a convective obstacle simulation with time-varying horizontal momentum extraction. Vertical momentum was extracted fully at all times. (a) Time variation of the phase parameter $\phi$ and fraction of extracted horizontal momentum $m_{u}$ (time is normalized by the reference time $\tau$ ). (b) Phase parameter $\phi$ as a function of extracted horizontal momentum $m_{u}$ showing irreversibility. Note that the hysteresis path is not a loop, since the system cannot leave the plume attractor once it has arrived there (see text).

Returning to the smaller systems of the present work, at Rayleigh numbers above $\sim 10^{5}$, the system exhibited oscillations in flow structure, wherein the convection plume periodically flipped from one side of the sink to the other (see Fig. 6 and animation: [53]). These oscillations only occurred in two cases: (1) when total momentum was extracted and (2) when vertical momentum was extracted and the system was in close proximity to the plume attractor (rather than the more common vortex attractor). When only horizontal momentum was extracted, oscillations did not occur due to the negligible interaction between flow and sink. Larger obstacles increased the magnitude of the fluctuations.

Although most oscillatory flows showed only a single characteristic frequency, more complex scenarios sometimes occurred, and it is highly likely that at even higher Rayleigh numbers, these oscillations will become larger in magnitude, 


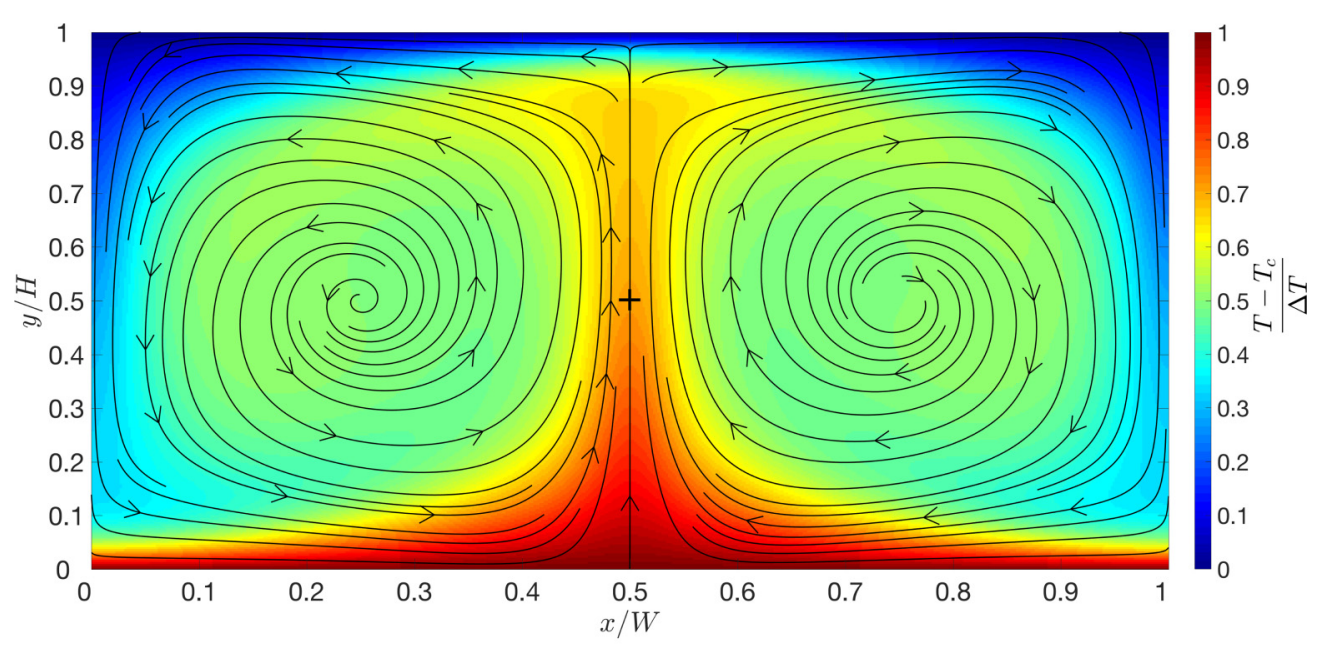

(a)

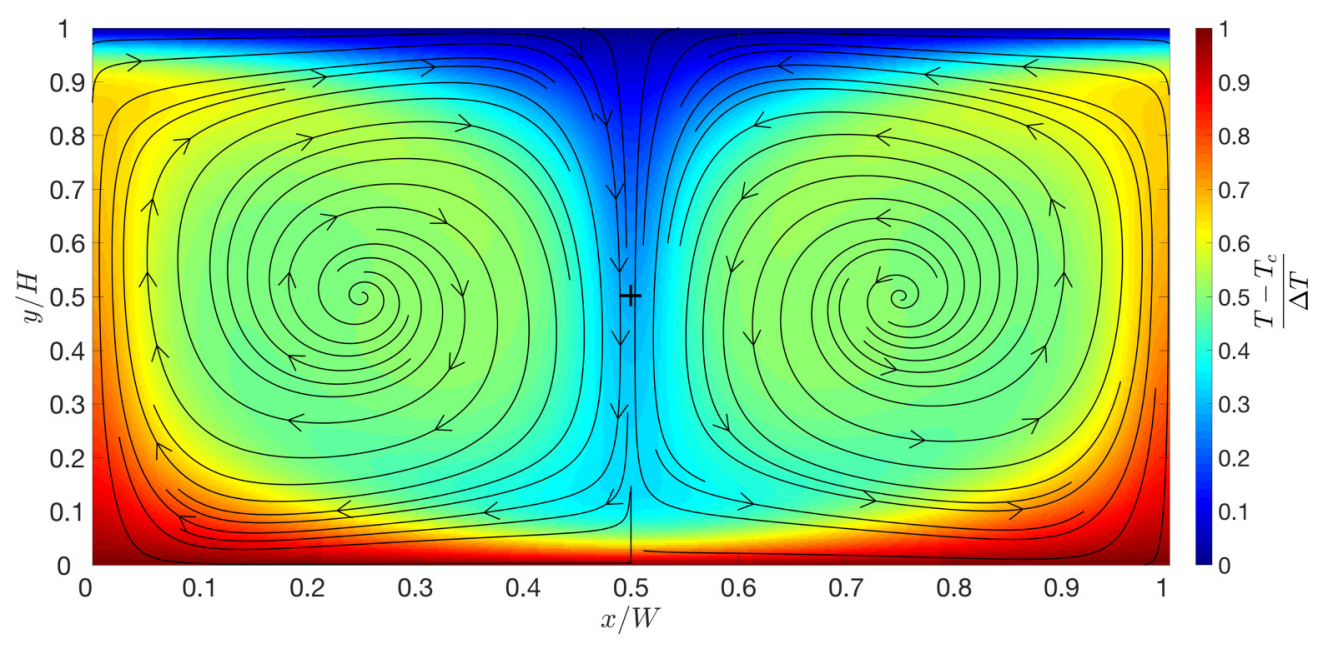

(b)

FIG. 9. The two stable attractors of a convective fluid driven at a Rayleigh number of $\mathrm{Ra}=10^{5}$ in the presence of a small sink of horizontal momentum placed at $[x, y]=[W / 2, H / 2]$ (the black crosshairs show its location; its size is only $\sim 0.7 \%$ of the system height). (a) Upwelling plume attractor. (b) Downwelling plume attractor.

and exhibit a more complex spectral distribution. The character of the oscillations also depended on initial conditions: some simulations with the same parameter values showed only weak oscillations, whereas others showed stronger fluctuations. This oscillatory dynamic highlighted the two conflicting physical imperatives in the system: hydrodynamic stability vs minimization of dissipation.

In terms of obstacle size, there was a clear trend of greater work extraction with smaller sink size. This is shown in Fig. 7, where the work output is plotted as a function of Rayleigh number for four different round obstacles (obstacle diameter is given as a fraction of system height). Smaller sinks produced less flow disruption and thus, even though they collected momentum from a smaller area, allowed more work to be extracted. Decreasing the obstacle size below one grid point was not possible, by nature of the simulation technique. However, it is expected that eventually the work production would peak and decline as obstacle size was decreased to extremely low values.

\section{BISTABILITY, HYSTERESIS, AND MEMORY}

We now turn to the bistability effect observed for systems with a sink of only vertical momentum. It was rare that the system settled upon the convection plume attractor spontaneously; however, it is possible to manipulate the system's energy landscape by varying the degree of momentum extraction. In the following, we conducted simulations with a single sink. At all times, this sink extracted all vertical momentum: $m_{v}=1$. The fraction of horizontal momentum extracted, $m_{u}$, was varied (see below). The Rayleigh number was also fixed at $\mathrm{Ra}=10^{4}$. The response of the system was measured using the phase parameter $\phi$ [see Eq. (4) and Fig. 2].

In the following hysteresis experiment, the variation of $m_{u}$ followed a ramp function as shown by the orange curve in Fig. 8(a). The system's response, measured by $\phi$, is shown by the small blue circles and blue curve in Fig. 8(a). Initially the system moves toward the vortex attractor with $\phi \rightarrow 0$, the more commonly observed attractor for vertical momentum 


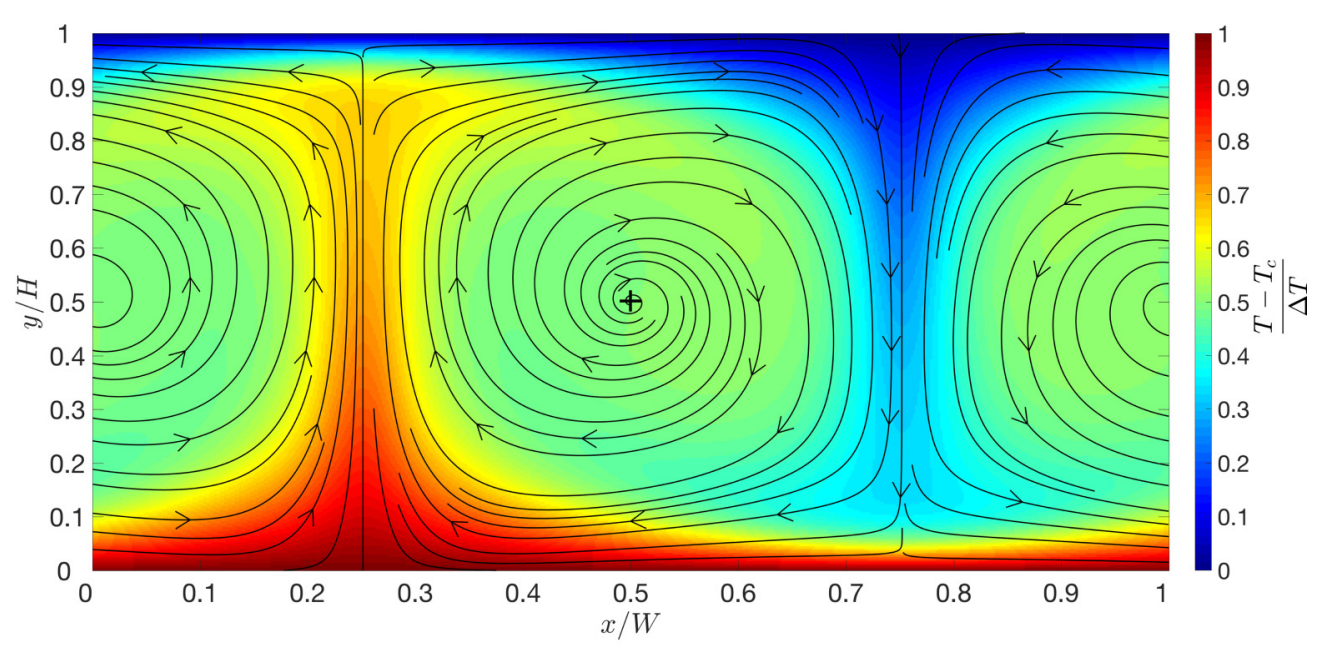

(a)

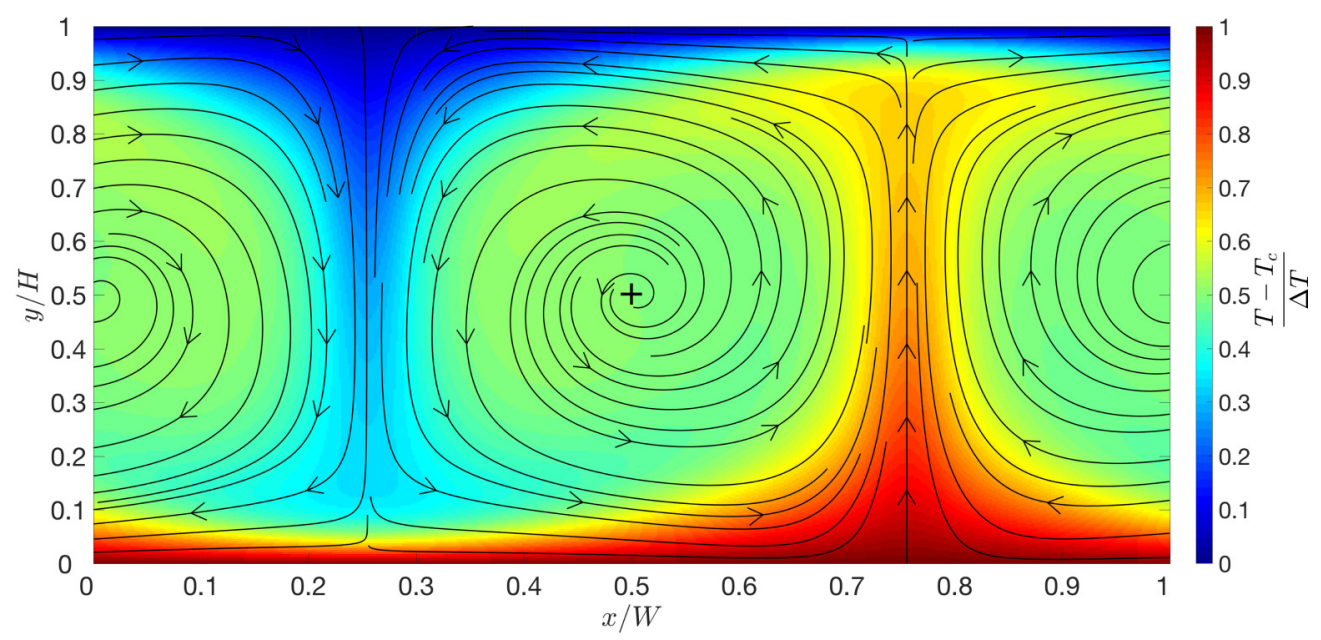

(b)

FIG. 10. The two most commonly occurring attractors of a convective fluid driven at a Rayleigh number of Ra $=10^{5}$ in the presence of a small sink of vertical momentum placed at $[x, y]=[W / 2, H / 2]$ (the black crosshairs show its location; its size is only $\sim 0.7 \%$ of the system height). (a) Clockwise vortex attractor. (b) Anticlockwise vortex attractor.

extraction. However, with increasing horizontal momentum extraction, the system moves toward the plume attractor with $\phi \rightarrow 0.25$. When horizontal momentum extraction was gradually returned to 0 , the system showed no response, and became trapped on the plume attractor, even when $m_{u}$ was held at 0 .

Figure $8(\mathrm{~b})$ shows the phase-space trajectory of this simulation. The vortex attractor (common for only vertical momentum extraction) is shown by the red circle and the plume attractor by the blue circle. This figure illustrates that the plume attractor is an absorbing state: once the system migrates there, it never leaves, even when $m_{u} \rightarrow 0$. This is why the hysteresis path actually fails to close. Once the system falls onto the plume attractor, there is no hydrodynamic driving force for migration back to the vortex attractor. The left-right symmetry of the flow field would first have to be broken for the plume to migrate away from the momentum sink. An animation of the simulation can be found at [54].

This experiment was repeated and similar behavior was observed in almost all cases. There were some cases where the system was drawn into the plume attractor from the beginning. This occurs if a plume happens to rise close to the sink as the convection pattern is first forming. We are currently in the process of quantifying the proximity with which a plume has to form to the sink in order for the system to migrate to the plume attractor.

This experiment highlights the ability of the system to store information about past states: when only vertical momentum is extracted, it is highly likely that the system will be found in the vortex attractor, but if horizontal momentum is extracted at any point, it becomes highly likely that the system will be absorbed into the plume attractor. Thus if we observed a system without knowing its parameter history, we could confidently predict whether or not it had ever been exposed to horizontal momentum extraction. The system thus stores a single bit of historical information about past states; it has a primitive memory. Inertial memory effects have been observed in the context of atmospheric convection [43], but not in a convective obstacle flow as presented here. Note that the spontaneous occurrence of the plume attractor in the pure 


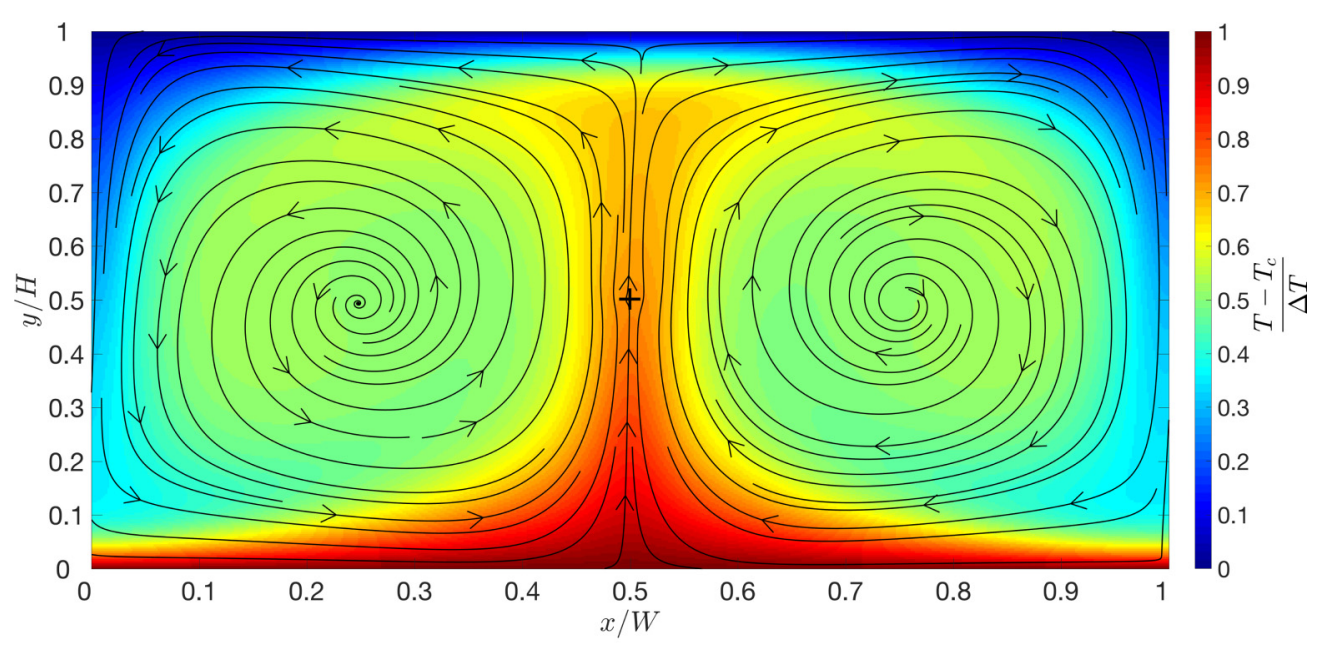

(a)

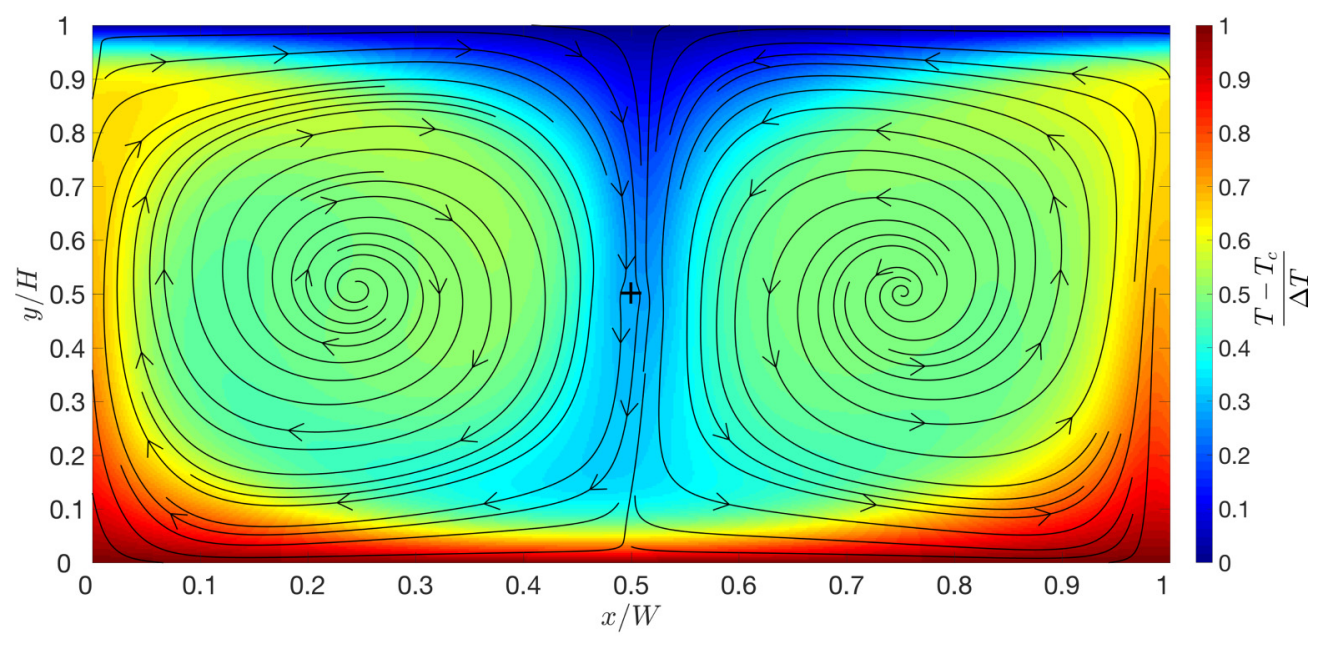

(b)

FIG. 11. The two less frequently occurring attractors of a convective fluid driven at a Rayleigh number of $\mathrm{Ra}=10^{5}$ in the presence of a small sink of vertical momentum placed at $[x, y]=[W / 2, H / 2]$ (the black crosshairs show its location; its size is only $\sim 0.7 \%$ of the system height). (a) Upwelling plume attractor. (b) Downwelling plume attractor. Note that these two attractors exhibit strong oscillations.

vertical sink case implies that this memory is not perfect, and we are in the process of quantifying the precision with which the system encodes its history.

The system presented above could be used as an OR gate from Boolean logic, wherein the two input bits are the presence (absence) of vertical and horizontal momentum extraction, and the output bit corresponds to whether the system lies in the plume attractor or not.

\section{CONCLUSIONS}

We have illustrated a curious and potentially useful subtlety of thermally driven convection systems. When only a single component of momentum is extracted by a small obstacle, the system maximizes dynamic stability and minimizes extracted work. But due to the inherent symmetries of convective flow fields, systems in which total momentum is extracted spontaneously maximize both their stability and extracted work. The system is also capable of encoding information about past states, due to a bistability that occurs when only vertical momentum is extracted. This vertical system yields two stable states: the vortex attractor and plume attractor. The plume attractor rarely occurs spontaneously, but if the system is manipulated with an adequate parameter protocol, it can be pushed into the shallow basin of attraction corresponding to this state. If the parameter protocol is instead unknown, the state of the system contains information about whether it was exposed to horizontal momentum extraction in its past.

Much of the research efforts in complexity science seek to understand the origins of adaptive behavior, agency, cognition, and evolution. In this paper we have illustrated bistability, memory, and elementary computation by an extremely simple physical system. The convection plumes in these systems originate from small fluctuations in an otherwise static, diffusive state. These fluctuations induce gravitational instabilities that translate into net forces on parcels of fluid. The resulting plumes accelerate exponentially until meeting the upper boundary of the system, whence they cool and may go on to establish stable convection cells. The additional component of finite obstacles induces a nontrivial symmetry- 


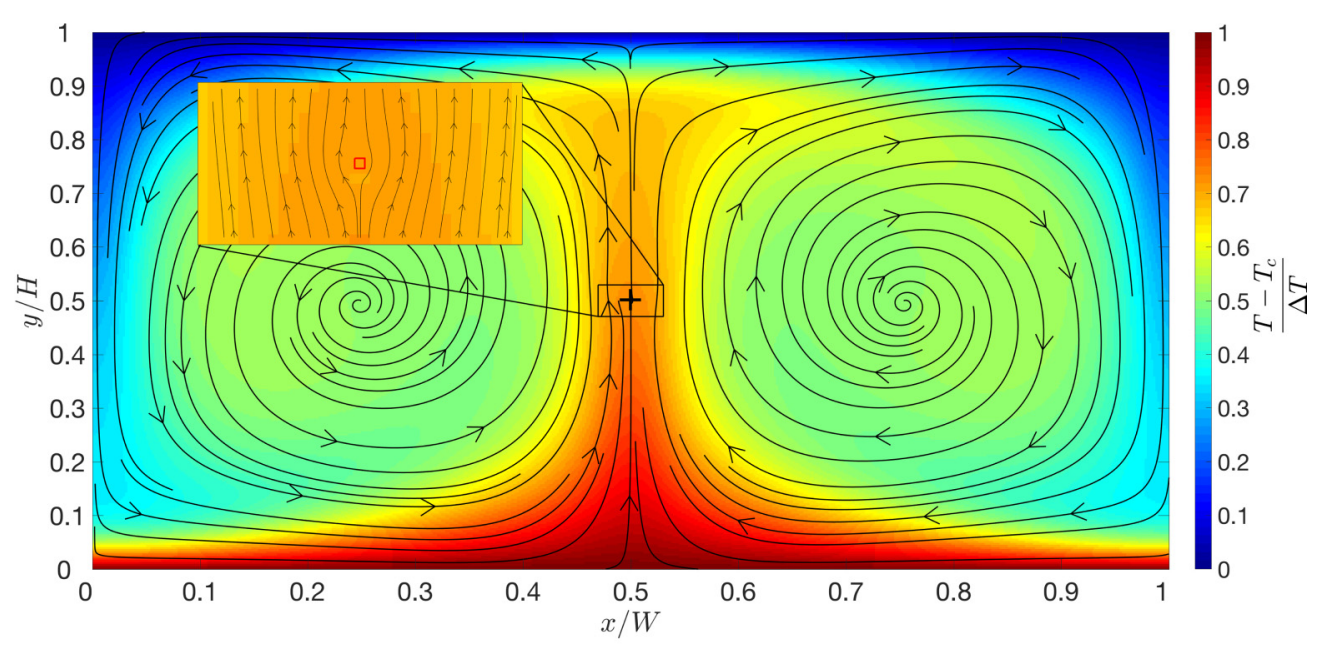

(a)

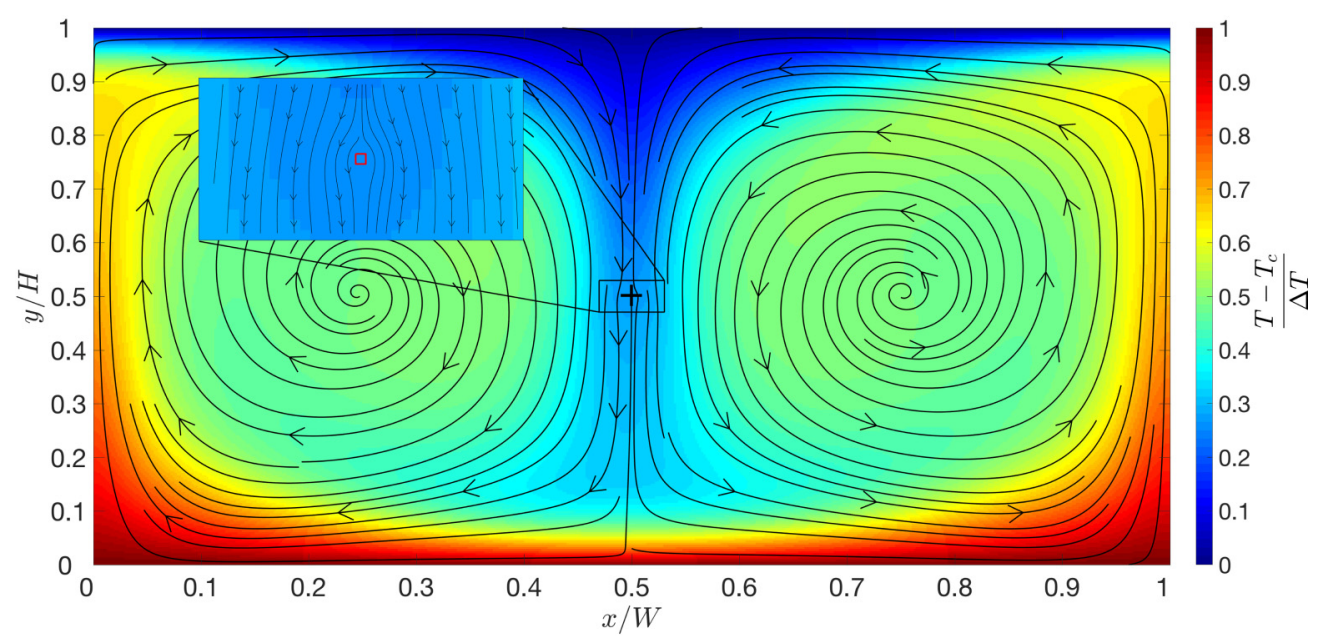

(b)

FIG. 12. The two stable attractors of a convective fluid driven at a Rayleigh number of Ra $=10^{5}$ in the presence of a small sink of total momentum placed at $[x, y]=[W / 2, H / 2]$ (the black crosshairs show its location; its size is only $\sim 0.7 \%$ of the system height). Inset figures show the local flow around the obstacle, with the red box indicating the area from which all momentum is extracted. (a) Upwelling plume attractor. (b) Downwelling plume attractor.

breaking effect (without such obstacles, convective fluids have horizontal translational symmetry). The existence of multiple attractors in the phase space of the vertical momentum sink system, permits a simple, single-bit memory, due to the plume state being an absorbing state. Furthermore, changes in the horizontal momentum extraction can be used to alter the attractor landscape. When the momentum extraction protocol is treated as an input, and the steady state of the system as output, the result is a fluid-based oR gate.

The field of computation by fluids [55-64] reaches back more than a century [65], from hydraulic root calculators [66,67], through fluidic logic in the 1960s [68], to microfluidic bubble logic in recent decades [69,70]. The bistability and attractor landscape of the system presented in this paper could easily be exploited for "convective computation," a concept that has never been advanced previously, despite the large range of devices in the field of fluid computation. This new form of fluid computation will be presented in forthcoming publications.
The phenomena of autocatalysis (during the initial growth of the convection plumes), negative feedback (the attractors are true attractors), memory, and simple computation are all present in this simple system. This suggests that such effects might be commonplace in a certain class of driven systems, especially when additional degrees of freedom and interactions, such as those of chemistry, are present. What remains to be elucidated are more precise necessary and sufficient conditions for such emergence.

\section{FUTURE WORK}

These simulations, while hydrodynamically accurate, omit some physical effects. Specifically, the model makes use of the Boussinesq approximation, and the fluid is assumed incompressible. Heat production and absorption due to expansion work is not included. We are currently incorporating such phenomena into our modeling framework, which will allow us to calculate real heat-to-work conversion efficiencies for 
convection, and thus give quantitative detail to speculations on the ability of convection to behave as a heat engine, and how close it can come to the Carnot limit.

In this work, simulations of turbulent convection flows were not performed. In this high Rayleigh number regime, it is possible that the system becomes less sensitive to the presence and symmetry-breaking influence of the momentum sink. Given the large, chaotic fluctuations that are characteristic of turbulence, it seems unlikely that memory and computation will still be viable: in turbulent flows information is generated at a higher rate than in laminar flows, but there is also an enhanced rate of information erasure due to irreversible, dissipative phenomena. It would, however, be informative to explore this regime in the future.

The ability to manipulate convection flows by altering momentum extraction from a small sink has potential applications in microfluidics, and any scenario where a convective flow needs to be controlled. As a final speculation, this attractive behavior of convection flows might have other practical relevance. Although solar-convective energy production was abandoned years ago, the fact that convection plumes are attracted to momentum sinks suggests a potential (though extremely difficult) means to artificially dissipate hurricanes and restrain their positions. One could envisage an array of extremely strong, biaxial offshore wind turbines, which could draw convective storm systems toward them. If the resistance of the turbines was appropriately controlled, it might be possible to gradually absorb kinetic energy from a storm until it was eventually dissipated. Such an approach has been considered previously, though without consideration of the effects presented in the present work [71].

\section{ACKNOWLEDGMENTS}

This work was supported by the Earth Life Science Institute Origins Network (EON) Postdoctoral Fellowship Program and the Caltech Division of Geological and Planetary Sciences Discovery Fund.

\section{APPENDIX: ATTRACTOR TYPES}

In the following figures we present all the different attractors of the $\mathrm{Ra}=10^{5}$ system. Figure 9 shows the two plume attractors for an obstacle that extracts only horizontal momentum extraction, Fig. 10 shows the more common vortex attractors for the vertical momentum extraction system, Fig. 11 shows the two less common plume attractors for the vertical momentum extraction system, and Fig. 12 shows the two plume attractors for the total momentum extraction system.
[1] B. Saltzman, Selected Papers on the Theory of Thermal Convection: With Special Application to the Earth's Planetary Atmosphere (Dover, New York, 1962).

[2] W. V. R. Malkus, Proc. R. Soc. London, Ser. A 225, 185 (1954).

[3] W. V. R. Malkus, Proc. R. Soc. London, Ser. A 225, 196 (1954).

[4] W. V. R. Malkus and G. Veronis, J. Fluid Mech. 4, 225 (1958).

[5] P. Manneville, Dynamics of Spatio-Temporal Cellular Structures (Springer, New York, 2006), pp. 41-65.

[6] G. W. Paltridge, Q. J. R. Meteorol. Soc. 104, 927 (1978).

[7] R. D. Lorenz, J. I. Lunine, P. G. Withers, and C. P. McKay, Geophys. Res. Lett. 28, 415 (2001).

[8] R. D. Lorenz, Int. J. Astrobiol 1, 3 (2002).

[9] R. D. Lorenz, Philos. Trans. R. Soc., B 365, 1349 (2010).

[10] R. Goody, J. Atmos. Sci. 64, 2735 (2007).

[11] R. Dewar, J. Phys. A: Math. Gen. 36, 631 (2003).

[12] R. Bradford, Phys. A (Amsterdam, Neth.) 392, 6273 (2013).

[13] T. Kita, J. Phys. Soc. Jpn. 75, 124005 (2006).

[14] H. Ozawa, S. Shimokawa, and H. Sakuma, Phys. Rev. E 64, 026303 (2001).

[15] S. Bartlett and S. Bullock, Phys. Rev. E 90, 023014 (2014).

[16] S. Bartlett and N. Virgo, Entropy 18, 431 (2016).

[17] Y. Kawazura and Z. Yoshida, Phys. Rev. E 82, 066403 (2010).

[18] Y. Kawazura and Z. Yoshida, Phys. Plasmas 19, 012305 (2012).

[19] Martin Mihelich, Davide Faranda, Didier Paillard, and Bérengère Dubrulle, Entropy 19, 154 (2017).

[20] I. Weaver, J. G. Dyke, and K. Oliver, in Beyond the Second Law: Entropy Production and Non-Equilibrium Systems, edited by R. C. Dewar, C. H. Lineweaver, R. K. Niven, and K. RegenauerLieb (Springer, Berlin, Heidelberg, 2014), pp. 277-290.
[21] G. E. Backus, Proc. Natl. Acad. Sci. USA 72, 1555 (1975).

[22] D. D. Koll and D. S. Abbot, Astrophys. J. 825, 99 (2016).

[23] D. D. Koll and T. D. Komacek, Astrophys. J. 853, 133 (2018).

[24] L. Michaud, Atmos. Res. 39, 157 (1995).

[25] Nilton O. Rennó and Andrew P. Ingersoll, J. Atmos. Sci. 53, 572 (1996).

[26] F. Stacey, Geophys. Surveys 3, 175 (1977).

[27] F. Stacey, Nature (London) 214, 476 (1967).

[28] O. R. Wulf and L. Davis Jr., J. Meteorol. 9, 80 (1952).

[29] M. Bister, N. Renno, O. Pauluis, and K. Emanuel, Proc. R. Soc. London, Ser. A 467, 1 (2011).

[30] A. M. Makarieva, V. G. Gorshkov, B.-L. Li, and A. D. Nobre, Proc. R. Soc. London, Ser. A 466, 1893 (2010).

[31] E. J. Braga and M. J. de Lemos, Int. Commun. Heat Mass Transfer 32, 1289 (2005).

[32] M. H. Esfe, A. A. A. Arani, A. Karimiopour, and S. S. M. Esforjani, Heat Transfer Res. 45, 279 (2014).

[33] M. Jami, A. Mezrhab, M. Bouzidi, and P. Lallemand, Int. J. Thermal Sci. 46, 38 (2007).

[34] Delphine Lacanette, Stéphane Vincent, Arthur Sarthou, Philippe Malaurent, and Jean-Paul Caltagirone, Int. J. Heat Mass Transfer 52, 2528 (2009).

[35] O. Laguerre, S. Benamara, D. Remy, and D. Flick, Int. J. Heat Mass Transfer 52, 5691 (2009).

[36] A. A. Mehrizi, M. Farhadi, H. H. Afroozi, K. Sedighi, and A. R. Darz, Int. Commun. Heat Mass Transfer 39, 1000 (2012).

[37] A. Merrikh, J. Enhanced Heat Transfer 8, 55 (2001).

[38] Ali A. Merrikh and José L. Lage, Int. J. Heat Mass Transfer 48, 1361 (2005). 
[39] M. Nazari, L. Louhghalam, and M. H. Kayhani, Chin. J. Chem. Eng. 23, 22 (2015).

[40] G. A. Sheikhzadeh, M. Nikfar, and A. Fattahi, J. Mech. Sci. Technol. 26, 3347 (2012).

[41] J. P. Crutchfield, W. L. Ditto, and S. Sinha, Chaos. 20, 037101 (2010).

[42] J. P. Crutchfield, Nat. Phys. 8, 17 (2012).

[43] L. Davies, R. S. Plant, and S. H. Derbyshire, J. Geophys. Res. 114, D17202 (2009).

[44] A. Brandstäter, J. Swift, Harry L. Swinney, A. Wolf, J. Doyne Farmer, Erica Jen, and P. J. Crutchfield, Phys. Rev. Lett. 51, 1442 (1983).

[45] D. A. Egolf, I. V. Melnikov, W. Pesch, and R. E. Ecke, Nature (London) 404, 733 (2000).

[46] A. Jayaraman, J. D. Scheel, H. S. Greenside, and P. F. Fischer, Phys. Rev. E 74, 016209 (2006).

[47] R. W. Walden, P. Kolodner, A. Passner, and C. M. Surko, Phys. Rev. Lett. 55, 496 (1985).

[48] S. Bartlett, Computation 5, 37 (2017).

[49] H. Dixit and V. Babu, Int. J. Heat Mass Transfer 49, 727 (2006).

[50] X. He, S. Chen, and G. D. Doolen, J. Comput. Phys. 146, 282 (1998).

[51] C.-H. Liu, K.-H. Lin, H.-C. Mai, and C.-A. Lin, Comput. Math. Appl. 59, 2178 (2010).

[52] Y. Peng, C. Shu, and Y. T. Chew, Phys. Rev. E 68, 026701 (2003).

[53] S. Bartlett, Convection with obstacle, $\mathrm{Ra}=2 \times 10^{5}$, https:// youtu.be/aY_YugztrrE, 2018.

[54] S. Bartlett, Convective obstacle flow hysteresis experiment, $\mathrm{Ra}=1 \times 10^{4}$, https://youtu.be/XuY8zi4jkAo, 2018.
[55] H. W. Avery, Fluid amplifier shift register circuit, US Patent No. 3,350,008 (1967).

[56] J. S. Chapline, Fluid shift register, US Patent No. 3,201,041 (1965).

[57] J. A. J. Gehring, J. Marvin, and R. T. Drake, Pure fluid computer, US Patent No. 3,190,554 (1965).

[58] C. M. Gobhai and J. E. Schoppe, Fluid logic arithmetic device, US Patent No. 3,243,114 (1966).

[59] G. N. Levesque and A. Hirt, Fluid logic circuit, US Patent No. 3,610,274 (1971).

[60] B. Peter, "and" gate, US Patent No. 3,191,611 (1965).

[61] R. E. Norwood, Fluid logic device, US Patent No. 3,128,040 (1964).

[62] R. E. Norwood, Fluid-operated logic devices, US Patent No. 3,318,329 (1967).

[63] E. R. Phillips, Pure fluid binary counter, US Patent No. 3,319,886 (1967).

[64] S. N. Zilberfarb, Pure fluid operated counter, US Patent No. 3,305,170 (1967).

[65] A. Adamatzky, arXiv:1811.09989.

[66] A. Emch, American Math. Mon. 8, 10 (1901).

[67] K. Foster and G. Parker, Fluidics: Components and circuits (Wiley-Interscience, Hoboken, 1970).

[68] M. Moylan, Fluid Logic in Simple Terms (Machinery publishing, Sussex, U.K., 1968).

[69] T. C. Draper, C. Fullarton, N. Phillips, B. P. de Lacy Costello, and A. Adamatzky, Sci. Rep. 8, 14153 (2018).

[70] M. Prakash and N. Gershenfeld, Science 315, 832 (2007).

[71] M. Z. Jacobson, C. L. Archer, and W. Kempton, Nat. Clim. Change 4, 195 (2014). 